\title{
Mitochondrial DNA polymorphisms and haplogroups in Parkinson's disease and control individuals with a similar genetic background
}

\author{
Helen Latsoudis · Cleanthe Spanaki · \\ Grigoris Chlouverakis · Andreas Plaitakis
}

Received: 3 November 2007 / Accepted: 22 January 2008/Published online: 20 February 2008

(C) The Japan Society of Human Genetics and Springer 2008

\begin{abstract}
Mitochondrial complex I deficiency has been implicated in the pathogenesis of Parkinson's disease (PD), but as yet no mitochondrial DNA (mtDNA) variations have been identified that could account for the impaired complex I activity. On the other hand, it has been suggested that mtDNA polymorphisms (mtSNPs) or haplogroups may modify the risk of developing PD. Here, we determined the distributions of ten mtSNPs that define the nine major European haplogroups among $224 \mathrm{PD}$ patients and 383 controls from Crete, an island of 0.6 million inhabitants who share a similar genetic background and a common environment. The recruitment of patients and controls was restricted to individuals of Cretan origin for at least three generations from both parental sides in order to avoid population admixture and subsequent genetic heterogeneity. We found no mtSNP or mtDNA haplogroup that predisposes to $\mathrm{PD}$, although there was a trend for haplogroups $\mathrm{J}, \mathrm{T}, \mathrm{U}$ and I and the supercluster of haplogroups UKJT to be slightly underrepresented in our PD patients as compared to controls. While a combination of common mtSNPs (present in $\geq 5 \%$ of the general population) may decrease the chance of developing PD, this effect was minor in the Cretan population.
\end{abstract}

Keywords mtDNA haplogroups $\cdot$ mtDNA polymorphisms · Parkinson's disease $\cdot$ Common environment $\cdot$ Similar genetic background

H. Latsoudis · C. Spanaki · A. Plaitakis $(\bowtie)$

Department of Neurology, University of Crete, Medical School,

Voutes and Staurakia, 71103 Heraklion, Crete, Greece

e-mail: plaitak@med.uoc.gr

G. Chlouverakis

Department of Biostatistics, University of Crete, Crete, Greece

\section{Introduction}

Parkinson's disease (PD) is a common neurodegenerative disorder affecting about $1 \%$ of the population at the age of 65. Although the symptomatology and neuropathologic features of the disorder have been well characterized, the underlying molecular mechanisms leading to this pathology are still obscure. The genetic hypothesis for PD has gained ground in recent years following the identification of six causative genes (SNCA, UCHL1, Parkin, DJ-1, PINK1 and LRRK2) and four putative loci (PARK3, PARK9, PARK10 and PARK11) linked to the rare familial forms of the disorder. These defects, however, are generally not present in the late-onset, mostly sporadic form of PD, which accounts for the overwhelming majority of cases that occur in the community.

While the etiology of PD remains unknown, several processes have been proposed in an attempt to unravel the mechanisms implicated in the neurodegeneration of nigral dopaminergic neurons associated with this disorder. These include mitochondrial dysfunction, protein misfolding, protein aggregation, oxidative stress, exogenous or endogenous neurotoxins and/or the interplay of genetic predisposition and environmental factors.

The possibility that mitochondrial dysfunction may play a role in PD was first raised by observations showing that $\mathrm{MPP}^{+}$, a neurotoxic agent used widely to produce an animal model for PD, inhibits complex I of the respiratory chain (Nicklas et al. 1987). Subsequent studies showing that certain pesticides (Sanchez-Ramos et al. 1998; Betarbet et al. 2000) or herbicides (Brooks et al. 1999) also capable of inhibiting complex I can produce animal models for PD have provided support for the environmental hypothesis for PD. Further studies using human tissues have shown that complex I activity is decreased in the 
platelets $(<50 \%)$ of PD subjects (Parker et al. 1989) and in substantia nigra (30-40\%) obtained at autopsy from PD patients (Schapira et al. 1990). Moreover, experiments on cytoplasmic hybrids derived from human neuroblastoma cells, which were depleted of their own mitochondrial DNA (mtDNA) and were repopulated with mitochondria from the platelets of PD patients, demonstrated that the complex I defect was exclusively transferred by patients' mtDNA (Swerdlow et al. 1996).

Although these observations have suggested that defects in complex I may play a role in the pathogenesis of PD, extensive sequencing of mtDNA in PD patients and controls has failed to identify mtDNA mutation(s) that could account for the described complex I reduction. Meanwhile, other investigators have suggested an association between a number of mitochondrial single nucleotide polymorphisms (mtSNPs) and the risk of developing PD. In this respect, several studies (Shoffner et al. 1993; Kösel et al. 1996; Egensperger et al. 1997; Otaegui et al. 2004) have shown that A4336G increases the risk for PD, whereas van der Walt et al. (2003) have presented evidence that A10398G exerts a protective effect against development of this disease. Furthermore, when data was stratified by gender and age, a protective effect was revealed for $9055 \mathrm{~A}$ and 13708A polymorphisms in female PD patients and cases older than 70 years of age, respectively. These results, however, have not been reproduced by others (Bandmann et al. 1997; Simon et al. 2000; Richter et al. 2002; Autere et al. 2004), who suggested that variations in the frequencies of mtSNPs could in fact reflect population stratification. Indeed, mtDNA sequence diversity has been shown to exist between populations with the 10398G polymorphism, for example, been shown to be present in $26 \%$ of white individuals, in $66 \%$ of Asians and in $91-96 \%$ of sub-Saharan Africans (Wallace et al. 1999).

More recently, a hypothesis has emerged that genetic variations of mtDNA could confer susceptibility to PD through a combination of polymorphisms rather than through the action of single sequence variants. MtDNA is a haploid nonrecombining genome characterized by a high mutation rate (van der Walt et al. 2003; Autere et al. 2004). Theoretically, polymorphisms within the four mitochondrial membrane-bound complexes (complex I-IV), as well as within the ATP6 gene encoding for the ATP synthase, may be of functional importance, since they may affect the production of ATP and radical oxygen species, which in turn could influence the risk of developing PD.

Various mtDNA polymorphisms are population-specific and are therefore clustered in lineages, with nine European mtDNA haplogroups ( H, I, J, K, T, U, V, W, and X) having been described (Wallace et al. 1999; Torroni et al. 1996; Finnilä et al. 2001; Herrnstadt et al. 2002). In keeping with this hypothesis, some investigators (van der Walt et al.
2003; Pyle et al. 2005) have found evidence that certain mtDNA haplogroups can modify the risk for PD. However, others (Ross et al. 2003; Autere et al. 2004) have been unable to replicate these results. It is presently unclear whether these discrepancies relate to stratification of the populations or to the size of the samples studied.

Here, we addressed this issue by studying mtDNA polymorphisms in PD patients and healthy subjects from Crete, a Greek island of 0.6 million inhabitants who have a similar genetic and cultural background and share a common environment. Until recently, the Cretan population had shown limited motility, and this has largely prevented the genetic admixture of the island's natives with other populations. To reduce the possibility of spurious findings resulting from population stratification, we included in this study only PD patients and controls whose ancestors from both parental lines were natives of Crete for at least three generations. In total, 383 healthy controls and 224 PD patients were genotyped for the ten mtDNA SNPs that define the nine major European mtDNA haplogroups. The two groups were assessed for the frequency of these SNPs and the distribution of their corresponding haplogroups and clusters of haplogroups. Moreover, the results obtained were compared with the distribution of mtDNA haplogroups in other European populations.

\section{Methods}

Patients and controls ascertainment

Patients were recruited from the outpatients clinic of the Neurology Department of the University Hospital of Crete during the period between 1997 and 2005. The diagnosis of PD was based on established criteria (Spanaki and Plaitakis 2004). The 224 PD patients studied here were selected from a cohort of 345 patients with Parkinsonism on the basis that they had typical PD. When PD was encountered in more than one family member, only the index case was included, as members of the same kindred may share genetic polymorphisms. The PD group consisted of 78 PD patients with positive family history (34.8\%) and 146 with the sporadic form of PD. The clinical and demographic characteristics of the PD patients have been reported previously (Spanaki and Plaitakis 2004).

Controls were randomly selected from the local population. Three hundred and eighty three individuals were recruited with a similar age and gender distribution to the patients' group and with no signs of neurodegenerative disorders prior to or during the study. Furthermore, we were careful to select individuals of Cretan origin going back at least three generations in order to avoid spurious associations due to variations in allelic frequencies in 
different populations. Both groups of patients and controls underwent a structured interview and were assessed for the presence of cognitive deficits with the mini mental state examination test (MMSE) (Folstein et al. 1975). Individuals who had MMSE scores of below 25 were excluded. Written consent was obtained from all participants according to the protocols approved by the institutional review board.

\section{Genotyping of European mtDNA haplogroups}

Genomic DNA was isolated from peripheral blood according to a standardised protocol. Oligonucleotide primers were designed to amplify mtDNA regions encompassing the ten polymorphic sites that define the major European mtDNA haplogroups (H, I, J, K, T, U, V, $\mathrm{W}, \mathrm{X})$. MtSNPs for typing were selected as previously described (Torroni et al. 1996; van der Walt et al. 2003) and mtDNAs were assigned to one of the nine major European haplogroups using RFLP analysis and the application of the SNaPshot (Applied Biosystems, Foster City, CA, USA) assay performed on an Applied Biosystems Prism $^{\circledR} 3100$ Genetic Analyser platform. The SNaPshot multiplex assay is a rapid screening technique based on the dideoxy single-base extension of unlabeled oligonucleotide primers (Brandstätter et al. 2003). Typing of mtSNPs using both techniques was performed blind to the clinical diagnosis of the samples. Primer sequences for PCR amplification and extension primers for SNaPshot assay are available upon request.

\section{Statistical analyses}

Departure from Hardy-Weinberg equilibrium was tested using a $\chi^{2}$ goodness of fit test as implemented in EPI-INFO 2005 (v. 3.3.2). Continuous (i.e., age) and categorical (i.e., gender) variables were compared between patients and controls using the independent samples $t$-test and Fisher's exact test, respectively. Frequencies of individual mtSNPs, haplogroups and clusters of haplogroups between cases and unaffected individuals were compared with the Fisher's exact or $\chi^{2}$ tests (SPSS, v. 14.0), as appropriate. Haplogroup clusters HV, KU, JT and IWX were constructed from phylogenetically closely related (i.e., on proximate branches) European mtDNA lineages (Richards et al. 1998). Risk of PD among members of different haplogroups or cluster of haplogroups was assessed by computing the odds ratios (OR) with their $95 \%$ confidence intervals $(95 \% \mathrm{CI})$. For these analyses each specific haplogroup and cluster of haplogroups was compared to all other haplogroups and clusters of haplogroups pooled into one group. All tests were performed at the two-sided 5\% level of significance.

\section{Results}

Our group of $224 \mathrm{PD}$ patients consisted of $55.4 \%$ males and $44.6 \%$ females with a mean age at disease onset of $63.1 \pm 10.8$ years and a mean age at examination of $71.5 \pm 9.2$ years. The mean age of the control group $(N=383)$ was $70.3 \pm 9.0$ years and composed of $54.3 \%$ males and $45.7 \%$ females. There were no significant differences in the age at examination $(P=0.13)$ and in the gender distribution (exact $P=0.87$ ) of our PD subjects and controls.

Analysis of the genotypic data revealed that the ten mtSNPs studied were comparably distributed between PD patients and controls (Table 1). A10398G, thought to lower the risk for PD, was present in $19.2 \%$ of our PD subjects and in $20.4 \%$ of our controls (OR 0.93 ; $95 \%$ CI $0.61-1.41$; $P=0.75$ ). Concerning the frequency of G9055A, which is thought to decrease PD risk in females, our data showed that this polymorphism was actually higher in our female PD cases $(8.0 \%)$ than in our female controls $(5.1 \%)$ (OR 0.62 ; 95\% CI 0.21-1.85, $P=0.49$ ), following stratification of the data by gender. As far as the G13708A polymorphism is concerned, 13708A was present in $7.9 \%$ of our PD patients older than 70 years of age and $11.3 \%$ in the younger PD cases (OR 0.67; 95\% CI 0.24-1.87; $P=0.47$ ). Similar frequencies were also obtained for the rest of the mtSNPs studied between patients and unaffected individuals following stratification by gender or age in both groups (data not shown).

The Cretan controls studied here displayed all nine major European haplogroups (H, I, J, K, T, U, V, W and X) (Table 2), with the frequencies of these mtDNA haplogroups being similar to those reported for other Southern European populations (Comas et al. 1997). These haplogroups were similarly distributed among our groups of PD cases and controls $\left(\chi^{2}=2.31 ; P=0.99\right)$, although there was a trend for haplogroups I, J, T and U to be slightly less frequent in the PD patients than in the controls. These differences were not statistically significant (Table 2) and remained nonsignificant following stratification of the data set by gender (data not shown), indicating a lack of a gender effect in our cohort of patients and controls. In addition, $3.9 \%$ of our controls and $5.8 \%$ of our PD cases (OR 1.51; 95\% CI $0.71-3.24 ; P=0.32$ ) could not be assigned to any of the previously characterized haplogroups (Torroni et al. 1996) and were classified here as "other."

Regarding the distributions of clusters or superclusters of mtDNA haplogroups, we found no significant 
Table 1 Distributions of allele frequencies of the ten mtSNPs that define the nine major European haplogroups between PD patients and controls

\begin{tabular}{|c|c|c|c|c|c|}
\hline & PD & Controls & $P$ & OR & $95.0 \% \mathrm{CI}$ \\
\hline \multicolumn{6}{|c|}{ G1719A } \\
\hline G & $207(92.4 \%)$ & $353(92.2 \%)$ & 0.999 & 0.966 & $0.520-1.795$ \\
\hline A & $17(7.6 \%)$ & $30(7.8 \%)$ & & & \\
\hline \multicolumn{6}{|c|}{ G4580A } \\
\hline G & $214(95.5 \%)$ & $367(95.8 \%)$ & 0.839 & 1.072 & $0.478-2.404$ \\
\hline A & $10(4.5 \%)$ & $16(4.2 \%)$ & & & \\
\hline \multicolumn{6}{|c|}{ C7028T } \\
\hline $\mathrm{C}$ & $118(52.7 \%)$ & $203(53 \%)$ & 0.999 & 1.013 & $0.728-1.410$ \\
\hline $\mathrm{T}$ & $106(47.3 \%)$ & $180(47 \%)$ & & & \\
\hline \multicolumn{6}{|c|}{ G8251A } \\
\hline G & $210(93.8 \%)$ & $357(93.2 \%)$ & 0.867 & 0.915 & $0.468-1.792$ \\
\hline A & $14(6.2 \%)$ & $26(6.8 \%)$ & & & \\
\hline \multicolumn{6}{|c|}{ G9055A } \\
\hline G & $210(93.8 \%)$ & $361(94.3 \%)$ & 0.859 & 1.094 & $0.548-2.184$ \\
\hline A & $14(6.2 \%)$ & $22(5.7 \%)$ & & & \\
\hline \multicolumn{6}{|c|}{ A10398G } \\
\hline A & $181(80.8 \%)$ & $305(79.6 \%)$ & 0.753 & 0.929 & $0.613-1.407$ \\
\hline G & $43(19.2 \%)$ & $78(20.4 \%)$ & & & \\
\hline \multicolumn{6}{|c|}{ A12308G } \\
\hline A & $185(82.6 \%)$ & $318(83 \%)$ & 0.911 & 1.031 & $0.667-1.596$ \\
\hline G & $39(17.4 \%)$ & $65(17 \%)$ & & & \\
\hline \multicolumn{6}{|c|}{ G13368A } \\
\hline G & $206(92 \%)$ & $348(90.9 \%)$ & 0.766 & 0.869 & $0.480-1.574$ \\
\hline A & $18(8 \%)$ & $35(9.1 \%)$ & & & \\
\hline \multicolumn{6}{|c|}{ G13708A } \\
\hline G & $204(91.1 \%)$ & $340(88.8 \%)$ & 0.410 & 0.775 & $0.444-1.355$ \\
\hline A & $20(8.9 \%)$ & $43(11.2 \%)$ & & & \\
\hline \multicolumn{6}{|c|}{ G16391A } \\
\hline G & $215(96 \%)$ & $363(94.8 \%)$ & 0.560 & 0.760 & $0.340-1.699$ \\
\hline A & $9(4 \%)$ & $20(5.2 \%)$ & & & \\
\hline
\end{tabular}

Odds ratios and their associated $95 \%$ confidence intervals were generated using Pearson's $\chi^{2}$ and Fisher's exact test in order to assess the risk of PD among individuals carrying a specific mtSNP

differences between our PD patients and the control groups (Table 3). Also, the resulting ORs indicated no significant differences in the risk of developing PD in members of a particular haplogroup or cluster of haplogroups (Fig. 1). However, there was a trend for the JT cluster to be less frequent in PD subjects (15.6\%) than in controls (18\%; OR $0.84 ; 95 \%$ CI $0.54-1.32 ; P=0.50$ ). In a similar manner, there was a trend for the frequencies of the UKJT and the JTIWX superclusters of haplogroups to be lower (by 6 and $10 \%$, respectively) in the PD patients than in the controls (Table 3).

\section{Discussion}

While the etiology of late-onset, mostly sporadic PD remains unknown, there is evidence that genetic predisposition, acting in concert with environmental factors, may be operational. However, it is presently unclear whether disease-predisposing variants are present in the mtDNA or in the nuclear DNA of susceptible individuals. In this regard, observations showing that environmental neurotoxins are capable of damaging the nigral cells, and thus affect cellular energy metabolism by inhibiting proteins involved in electron transfer, have suggested that mitochondrial dysfunction is involved in PD pathogenesis. In accord with this possibility are observations showing that wild-type parkin (Darios et al. 2003), DJ-1 (Canet-Avilés et al. 2004) and PINK1 (Clark et al. 2006), three proteins implicated in the monogenetic forms of PD, play a role in maintaining mitochondrial integrity. Also, factors thought to contribute to the nigral degeneration such as oxidative stress, $S$-nitrosylation and dopamine, are shown to compromise parkin function (Chung et al. 2004; Yao et al. 2004; LaVoie et al. 2005).

Here, we tested whether certain polymorphisms in mtDNA can predispose to PD in a population that is rather genetically homogeneous, as the interaction of a genetic variant with a particular disease is rather likely when this occurs across populations. A cohort of 224 native PD patients and 383 unaffected individuals from the island of Crete were genotyped for the ten mtSNPs that define the nine European mtDNA haplogroups. The distributions of these mtSNPs, the constructed haplogroups and the clusters or superclusters of these haplogroups among PD and control subjects were determined.

Of the SNPs studied here, the 10398G shows nonspecific geographic prevalence in European ( $\mathrm{J}, \mathrm{K}$, and $\mathrm{I}$ ), Asian (C, D, E, M) and African (L) haplogroups (Herrnstadt et al. 2002), probably suggesting that it arose through independent mutational events during the migration of ancient humans out of Africa. On the other hand, it appears that haplogroup $\mathrm{J}$ and the phylogenetically related haplogroup $\mathrm{T}$ were derived from a common ancestral JT haplogroup following a mutational event that probably took place in the mtDNA of a woman that lived in the Anatolian-Caucasus region around 45,000 years bp. Both haplogroups are associated with the spread of farming and herding in Europe during the Neolithic era $(8,000$ 10,000 years bp). Haplogroup J, but not T, is defined by the presence of $10398 \mathrm{G}$, mentioned above, that causes a nonconservative substitution of alanine for threonine within the NADH dehydrogenase (ND3) subunit of complex I (van der Walt et al. 2003). This particular polymorphism is also shared by haplogroups $\mathrm{K}$ and I that are not phylogenetically related (Torroni et al. 1996; Wallace et al. 1999). 


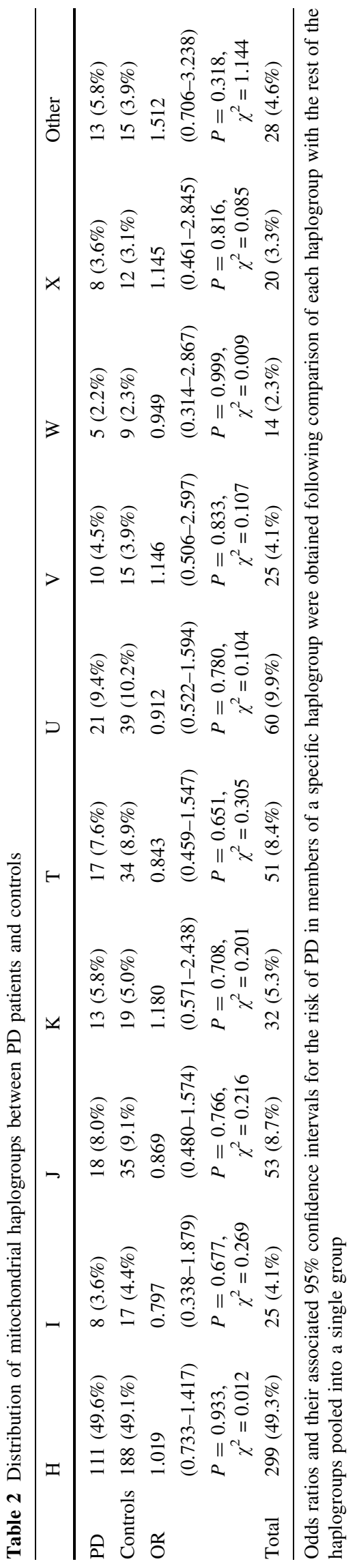

Results from the present study, the first to evaluate the frequencies of mtDNA polymorphisms and their corresponding haplogroups in the Greek population, revealed that these frequencies fall within the range of previously published values for Europeans (Torroni et al. 1996; Comas et al. 1997). Specifically, our results indicated that the distributions of the nine major European haplogroups among Cretans did not differ significantly from those of the surrounding SW European genetic landscape, i.e., Sicilians, Sardinians, South and Central Italians, Andalusians, Catalans, Central Spaniards, etc., supporting the hypothesis of a homogeneous mitochondrial genetic flow in countries of the European Mediterranean basin (Plaza et al. 2003; Achilli et al. 2004).

The present data did not reveal any significant differences in the distributions of the ten mtSNPs studied among our PD patients and controls. Specifically, our finding that 10398G (ND3 polymorphism) does not alter significantly the risk for PD in the Cretan population is in keeping with previous studies (Bandmann et al. 1997; Simon et al. 2000; Richter et al. 2002; Otaegui et al. 2004; Autere et al. 2004; Ghezzi et al. 2005). Similarly, polymorphisms within the ND2 (i.e., G4580A) and the ND5 (i.e., G13368A and G13708A) subunits of complex I did not interact with the chance of developing PD in our population. This was also true for mtSNPs within the COI or cytochrome c oxidase I (C7028T) and COII (G8251A) of complex IV as well as for ATP6 (G9055A) of ATP synthase (for some complex $\mathrm{V})$. Moreover, our data showing a trend for a higher frequency of 9055A (within the ATP6 gene) in female PD patients than in female controls is not in agreement with a protective effect as previously suggested (van der Walt et al. 2003; Huerta et al. 2005). However, we observed a trend for a decreased PD risk regarding 13708A allele (within the ND5 subunit) in individuals older than 70 years following the stratification of the dataset by age, in accordance with the data from van der Walt et al. (2003).

Regarding the possible interaction of mtDNA haplogroups with PD, our data revealed a trend for haplogroups $\mathrm{I}, \mathrm{J}, \mathrm{T}$ and $\mathrm{U}$ to be underrepresented in the PD group. These results tend to support previous observations showing that haplogroups J (van der Walt et al. 2003; Pyle et al. 2005) and U (Pyle et al. 2005) confer protection against PD. Our findings showing a trend for haplogroup $\mathrm{K}$ to be slightly increased in PD patients (OR 1.18; 95\% CI 0.57-2.44; $P=0.71)$ do not support a suggested protective effect for this haplogroup (van der Walt et al. 2003; Ghezzi et al. 2005).

These discrepancies may be due to the fact that mtDNA-haplogroup association studies are prone to methodological biases (Ioannidis 2003; Raule et al. 2007), mostly because mtDNA sequence diversity exists between populations. Hence it is not surprising that inconsistent 
Table 3 Distribution of mitochondrial haplogroup clusters and superclusters between PD patients and controls

\begin{tabular}{lllllllll}
\hline & HV & KU & JT & IWX & HVKU & JTIWX & UKJT & HVIWX \\
\hline PD & $121(54 \%)$ & $34(15.2 \%)$ & $35(15.6 \%)$ & $21(9.4 \%)$ & $155(69.2 \%)$ & $56(25 \%)$ & $69(30.8 \%)$ & $142(63.4 \%)$ \\
controls & $203(53 \%)$ & $58(15.1 \%)$ & $69(18 \%)$ & $38(9.9 \%)$ & $261(68.1 \%)$ & $107(27.9 \%)$ & $127(33.2 \%)$ & $241(62.9 \%)$ \\
OR & 1.042 & 1.003 & 0.843 & 0.939 & 1.050 & 0.860 & 0.897 & 1.020 \\
& $(0.748-1.450)$ & $(0.633-1.588)$ & $(0.540-1.315)$ & $(0.536-1.645)$ & $(0.736-1.499)$ & $(0.590-1.252)$ & $(0.629-1.279)$ & $(0.725-1.436)$ \\
& $P=0.866$, & $P=0.999$, & $P=0.504$, & $P=0.888$, & $P=0.856$, & $P=0.449$, & $P=0.590$, & $P=0.931$, \\
& $\chi^{2}=0.059$ & $\chi^{2}=0.000$ & $\chi^{2}=0.569$ & $\chi^{2}=0.048$ & $\chi^{2}=0.072$ & $\chi^{2}=0.621$ & $\chi^{2}=0.359$ & $\chi^{2}=0.013$ \\
Total & $324(53.4 \%)$ & $92(15.2 \%)$ & $104(17.1 \%)$ & $59(9.7 \%)$ & $416(68.5 \%)$ & $163(26.9 \%)$ & $196(32.3 \%)$ & $383(63.1 \%)$ \\
\hline
\end{tabular}

Odds ratios and their associated 95\% confidence intervals for the risk of PD in members of a specific haplogroup cluster were obtained following comparison of each cluster with the other haplogroup clusters pooled into a single group

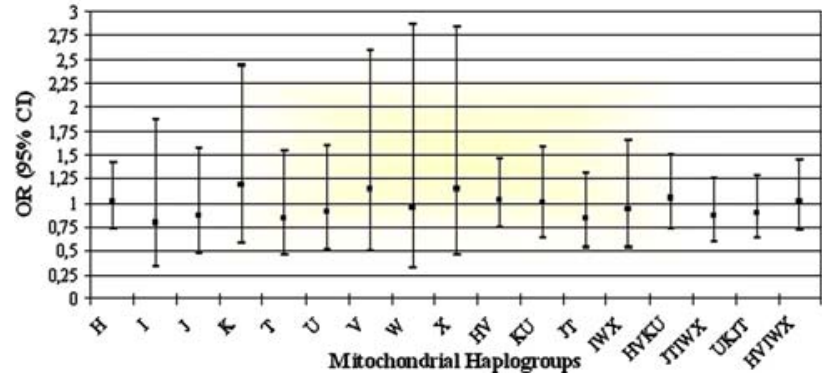

Fig. 1 Odds ratios and their associated 95\% confidence intervals used to assess the risk of PD in members of a specific haplogroup or cluster of haplogroups were generated following comparison of each specific haplogroup with the others (pooled into a single group)

results characterize studies, even those with relatively large sample sizes (van der Walt et al. 2003; Pyle et al. 2005; Ghezzi et al. 2005), regarding the effect of various haplogroups (i.e., $\mathrm{K}$ or $\mathrm{U}$ ) on protection from the disease. Here, in order to reduce the possibility of mtDNA heterogeneity resulting from the genetic admixture of people with different ethnic backgrounds, we restricted our study to individuals of Cretan origin stretching back at least three generations from both the maternal and paternal side. Furthermore, we recruited PD patients and control individuals randomly from various parts of the island in order to reduce the risk of bias due to the founder's effect, as seen in small locales.

With respect to the effect of clusters or superclusters of mtDNA haplogroups, our data showed a trend for superclusters UKJT and JTIWX to be underrepresented in PD patients. On the other hand, supercluster HVKU was slightly more frequent in affected individuals than in controls. As such, our findings showing a trend for supercluster UKJT to be slightly decreased in PD patients tend to support the suggestion by Pyle et al. (2005) regarding the protective effect of this supercluster. However, we found no evidence that the presence of a JT cluster or a JTIWX supercluster constitutes risk factors for PD, as has been previously suggested by Ross et al. (2003) or Autere et al. (2004), respectively.

From the present data, it can be deduced that no mtDNA variations predisposing to $\mathrm{PD}$ are present in the Cretan population, and that these results are in keeping with previous studies that failed to detect causally related mtDNA sequence variations in this disorder. On the other hand, we have detected a trend for haplogroups I, J, T and $\mathrm{U}$ and for supercluster UKJT to be less frequent in PD patients than in controls. As most of the mtSNPs studied here are common in the general Cretan population, our findings are in agreement with the view (Ioannidis et al. 2006; Topol and Frazer 2007) that the influence of genetic variants present in $\geq 5 \%$ of the general population on complex disorders are generally small (ORs between 1.2 and 1.6). In accordance with this notion, genome-wide association studies (Fung et al. 2006; Elbaz et al. 2006) have also shown that common genetic polymorphisms have a minor or moderate effect on the risk for PD. Evidently, the detection of such minor effects requires very large sample sizes. Hence, the results of the present study are consistent with the hypothesis that possessing a combination of certain mtDNA polymorphisms may decrease the chance of developing PD, but this protective effect was minor in the island population studied.

Acknowledgments We are deeply indebted to the patients and family members who participated in this study. We also thank Irene Skoula for an excellent technical support and the students of the Master's of Neuroscience course for their invaluable contributions. H.L. and C.S. were supported by the Association for Research and Treatment of Neurologic Disorders of Crete ("Ev Z $\eta v$ ") and by the Second Operational Programme for Education and Initial Vocational Training (EPEAEK II) from the Ministry of Education of Greece.

\section{References}

Achilli A, Rengo C, Magri C, Battaglia V, Olivieri A, Scozzari R, Cruciani F, Zeviani M, Briem E, Carelli V, Moral P, Dugoujon JM, Roostalu U, Loogväli EL, Kivisild T, Bandelt HJ, Richards M, Villems R, Santachiara-Benerecetti AS, Semino O, Torroni A 
(2004) The molecular dissection of mtDNA haplogroup $\mathrm{H}$ confirms that the Franco-Cantabrian glacial refuge was a major source for the European gene pool. Am J Hum Genet 75(5): 910-918

Autere J, Moilanen JS, Finnilä S, Soininen H, Mannermaa A, Hartikainen P, Hallikainen M, Majamaa K (2004) Mitochondrial DNA polymorphisms as risk factors for Parkinson's disease and Parkinson's disease dementia. Hum Genet 115(1):29-35

Bandmann O, Sweeney MG, Daniel SE, Marsden CD, Wood NW (1997) Mitochondrial DNA polymorphisms in pathologically proven Parkinson's disease. J Neurol 244(4):262-265

Betarbet R., Sherer TB, MacKenzie G, Garcia-Osuna M, Panov AV, Greenamyre JT (2000) Chronic systemic pesticide exposure reproduces features of Parkinson's disease. Nat Neurosci 3:1301-1306

Brandstätter A, Parsons TJ, Parson W (2003) Rapid screening of mtDNA coding region SNPs for the identification of west European Caucasian haplogroups. Int J Legal Med 117(5): 291-298

Brooks AI, Chadwick CA, Gelbard HA, Cory-Slechta DA, Federoff HJ (1999) Paraquat elicited neurobehavioral syndrome caused by dopaminergic neuron loss. Brain Res 823:1-10

Canet-Avilés RM, Wilson MA, Miller DW, Ahmad R, McLendon C, Bandyopadhyay S, Baptista MJ, Ringe D, Petsko GA, Cookson MR (2004) The Parkinson's disease protein DJ-1 is neuroprotective due to cysteine-sulfinic acid-driven mitochondrial localization. Proc Natl Acad Sci USA 101(24):9103-9108

Chung KK, Thomas B, Li X, Pletnikova O, Troncoso JC, Marsh L, Dawson VL, Dawson TM (2004) S-nitrosylation of parkin regulates ubiquitination and compromises parkin's protective function. Science 304(5675):1328-1331

Clark IE, Dodson MW, Jiang C, Cao JH, Huh JR, Seol JH, Yoo SJ, Hay BA, Guo M (2006) Drosophila pink1 is required for mitochondrial function and interacts genetically with parkin. Nature 441(7097):1162-1166

Comas D, Calafell F, Mateu E, Pérez-Lezaun A, Bosch E, Bertranpetit J (1997) Mitochondrial DNA variation and the origin of the Europeans. Hum Genet 99(4):443-449

Darios F, Corti O, Lücking CB, Hampe C, Muriel MP, Abbas N, Gu WJ, Hirsch EC, Rooney T, Ruberg M, Brice A (2003) Parkin prevents mitochondrial swelling and cytochrome $\mathrm{c}$ release in mitochondria-dependent cell death. Hum Mol Genet 12(5): $517-526$

Egensperger R, Kösel S, Schnopp NM, Mehraein P, Graeber MB (1997) Association of the mitochondrial tRNA (A4336G) mutation with Alzheimer's and Parkinson's diseases. Neuropathol Appl Neurobiol 23(4):315-321

Elbaz A, Nelson LM, Payami H, Ioannidis JP, Fiske BK, Annesi G, Carmine Belin A, Factor SA, Ferrarese C, Hadjigeorgiou GM, Higgins DS, Kawakami H, Krüger R, Marder KS, Mayeux RP, Mellick GD, Nutt JG, Ritz B, Samii A, Tanner CM, Van Broeckhoven C, Van Den Eeden SK, Wirdefeldt K, Zabetian CP, Dehem M, Montimurro JS, Southwick A, Myers RM, Trikalinos TA (2006) Lack of replication of thirteen single-nucleotide polymorphisms implicated in Parkinson's disease: a large-scale international study. Lancet Neurol 5(11):917-923

Finnilä S, Lehtonen MS, Majamaa K (2001) Phylogenetic network for European mtDNA. Am J Hum Genet 68(6):1475-1484

Folstein MF, Folstein SE, McHugh PR (1975) "Mini-mental state". A practical method for grading the cognitive state of patients for the clinician. J Psychiatr Res 12(3):189-198

Fung HC, Scholz S, Matarin M, Simón-Sánchez J, Hernandez D, Britton A, Gibbs JR, Langefeld C, Stiegert ML, Schymick J, Okun MS, Mandel RJ, Fernandez HH, Foote KD, Rodríguez RL, Peckham E, De Vrieze FW, Gwinn-Hardy K, Hardy JA, Singleton A (2006) Genome-wide genotyping in Parkinson's disease and neurologically normal controls: first stage analysis and public release of data. Lancet Neurol 5(11):911-916

Ghezzi D, Marelli C, Achilli A, Goldwurm S, Pezzoli G, Barone P, Pellecchia MT, Stanzione P, Brusa L, Bentivoglio AR, Bonuccelli U, Petrozzi L, Abbruzzese G, Marchese R, Cortelli P, Grimaldi D, Martinelli P, Ferrarese C, Garavaglia B, Sangiorgi S, Carelli V, Torroni A, Albanese A, Zeviani M (2005) Mitochondrial DNA haplogroup $\mathrm{K}$ is associated with a lower risk of Parkinson's disease in Italians. Eur J Hum Genet 13(6): 748-752

Herrnstadt C, Elson JL, Fahy E, Preston G, Turnbull DM, Anderson C, Ghosh SS, Olefsky JM, Beal MF, Davis RE, Howell N (2002) Reduced-median-network analysis of complete mitochondrial DNA coding-region sequences for the major African, Asian, and European haplogroups. Am J Hum Genet 70(5): $1152-1171$

Huerta C, Castro MG, Coto E, Blázquez M, Ribacoba R, Guisasola LM, Salvador C, Martínez C, Lahoz CH, Alvarez V (2005) Mitochondrial DNA polymorphisms and risk of Parkinson's disease in Spanish population. J Neurol Sci 236(1-2):49-54

Ioannidis JP (2003) Genetic associations: false or true? Trends Mol Med 9(4):135-138

Ioannidis JP, Trikalinos TA, Khoury MJ (2006) Implications of small effect sizes of individual genetic variants on the design and interpretation of genetic association studies of complex diseases. Am J Epidemiol 164(7):609-614

Kösel S, Lücking CB, Egensperger R, Mehraein P, Graeber MB (1996) Mitochondrial NADH dehydrogenase and CYP2D6 genotypes in Lewy-body parkinsonism. J Neurosci Res 44(2):174-183

LaVoie MJ, Ostaszewski BL, Weihofen A, Schlossmacher MG, Selkoe DJ (2005) Dopamine covalently modifies and functionally inactivates parkin. Nat Med 11(11):1214-1221

Nicklas WJ, Youngster SK, Kindt MV, Heikkila RE (1987) MPTP, MPP+ and mitochondrial function. Life Sci 40(8):721-729

Otaegui D, Paisán C, Sáenz A, Martí I, Ribate M, Martí-Massó JF, Pérez-Tur J, López de Munain A (2004) Mitochondrial polymorphisms in Parkinson's Disease. Neurosci Lett 370(2-3): $171-174$

Parker WD Jr, Boyson SJ, Parks JK (1989) Abnormalities of the electron transport chain in idiopathic Parkinson's disease. Ann Neurol 26(6):719-723

Plaza S, Calafell F, Helal A, Bouzerna N, Lefranc G, Bertranpetit J, Comas D (2003) Joining the pillars of Hercules: mtDNA sequences show multidirectional gene flow in the western Mediterranean. Ann Hum Genet 67(Pt 4):312-328

Pyle A, Foltynie T, Tiangyou W, Lambert C, Keers SM, Allcock LM, Davison J, Lewis SJ, Perry RH, Barker R, Burn DJ, Chinnery PF (2005) Mitochondrial DNA haplogroup cluster UKJT reduces the risk of PD. Ann Neurol 57(4):564-567

Raule N, Sevini F, Santoro A, Altilia S, Franceschi C (2007) Association studies on human mitochondrial DNA: methodological aspects and results in the most common age-related diseases. Mitochondrion 7(1-2):29-38

Richards MB, Macaulay VA, Bandelt HJ, Sykes BC (1998) Phylogeography of mitochondrial DNA in western Europe. Ann Hum Genet 62(Pt 3):241-260

Richter G, Sonnenschein A, Grünewald T, Reichmann H, Janetzky B (2002) Novel mitochondrial DNA mutations in Parkinson's disease. J Neural Transm 109(5-6):721-729

Ross OA, McCormack R, Maxwell LD, Duguid RA, Quinn DJ, Barnett YA, Rea IM, El-Agnaf OM, Gibson JM, Wallace A, Middleton D, Curran MD (2003) mt $4216 \mathrm{C}$ variant in linkage with the mtDNA TJ cluster may confer a susceptibility to mitochondrial dysfunction resulting in an increased risk of Parkinson's disease in the Irish. Exp Gerontol 38(4):397-405 
Sanchez-Ramos J, Facca A, Basit A, Song S (1998) Toxicity of dieldrin for dopaminergic neurons in mesencephalic cultures. Exp Neurol 150:263-271

Schapira AH, Mann VM, Cooper JM, Dexter D, Daniel SE, Jenner P, Clark JB, Marsden CD (1990) Anatomic and disease specificity of NADH CoQ1 reductase (complex I) deficiency in Parkinson's disease. J Neurochem 55:2142-2145

Shoffner JM, Brown MD, Torroni A, Lott MT, Cabell MF, Mirra SS, Beal MF, Yang CC, Gearing M, Salvo R, et al. (1993) Mitochondrial DNA variants observed in Alzheimer disease and Parkinson disease patients. Genomics 17(1):171-184

Simon DK, Mayeux R, Marder K, Kowall NW, Beal MF, Johns DR (2000) Mitochondrial DNA mutations in complex I and tRNA genes in Parkinson's disease. Neurology 54(3):703-709

Spanaki C, Plaitakis A (2004) Bilineal transmission of Parkinson disease on Crete suggests a complex inheritance. Neurology 62(5):815-817

Swerdlow RH, Parks JK, Miller SW, Tuttle JB, Trimmer PA, Sheehan JP, Bennett JP Jr, Davis RE, Parker WD Jr (1996) Origin and functional consequences of the complex I defect in Parkinson's disease. Ann Neurol 40(4):663-71
Topol EJ, Frazer KA (2007) The resequencing imperative. Nat Genet 39(4):439-440

Torroni A, Huoponen K, Francalacci P, Petrozzi M, Morelli L, Scozzari R, Obinu D, Savontaus ML, Wallace DC (1996) Classification of European mtDNAs from an analysis of three European populations. Genetics 144(4):1835-1850

van der Walt JM, Nicodemus KK, Martin ER, Scott WK, Nance MA, Watts RL, Hubble JP, Haines JL, Koller WC, Lyons K, Pahwa R, Stern MB, Colcher A, Hiner BC, Jankovic J, Ondo WG, Allen FH Jr, Goetz CG, Small GW, Mastaglia F, Stajich JM, McLaurin AC, Middleton LT, Scott BL, Schmechel DE, Pericak-Vance MA, Vance JM (2003) Mitochondrial polymorphisms significantly reduce the risk of Parkinson disease. Am J Hum Genet 72(4):804-811

Wallace DC, Brown MD, Lott MT (1999) Mitochondrial DNA variation in human evolution and disease. Gene 238(1):211-230

Yao D, Gu Z, Nakamura T, Shi ZQ, Ma Y, Gaston B, Palmer LA, Rockenstein EM, Zhang Z, Masliah E, Uehara T, Lipton SA (2004) Nitrosative stress linked to sporadic Parkinson's disease: $S$-nitrosylation of parkin regulates its E3 ubiquitin ligase activity. Proc Natl Acad Sci USA 101(29):10810-10814 